\title{
Blockchain-based Power Trading Process
}

\author{
You-Jin Song \\ Dongguk University at Gyeongju Campus, Korea \\ song@dongguk.ac.kr
}

\begin{abstract}
Recently, the paradigm of the power industry has been digitalization with a focus on renewable energy. With this shift toward the energy-ICT convergence accelerator, there will also be many changes in effect energy policies. In particular, in terms of energy demand management, there will be regional-centric, self-reliant decentralization, and the activation of Distributed Energy Resources (DERs) including renewable energy will result in the deployment of microgrid-type virtual power plants on a region-wide basis. This paper designs a blockchain-based power transaction process in which individuals (producers) can produce and use power themselves or sell the remaining power to others, rather than transmitting and using the developed power from the existing centralized power.
\end{abstract}

Keywords: Internet of things, Blockchain, Smart contract, Power trading

\section{Introduction}

Recently, People-to-People (P2P), people-to-machine (P2M), and machine-to-machine (M2M) through digital and Information and Communication Technology (ICT) are entering a hyperconnected society that is closely connected across online and offline. A hyper-connected society refers to a society where people, processes, data and objects can be linked together to create new values and innovations through intelligent networks. In particular, ICT applications in the power industry can lead to the efficient operation of existing energy systems, as well as the creation of new values such as the combination of renewable energy support and power storage devices, electric vehicles and the development of various energy services.

The energy sector is also expected to accelerate the transformation of the energy system and contribute to a drastic change in daily life through the fusion of technical elements of the fourth industrial revolution under the smart grid base centered on the power industry. Energy storage technology, combined with small distributed energy resources, plays an important role in system-linked or standalone operations such as microgrids and virtual power plants. Integrated operation of microgrids, virtual power plants and energy management systems (EMS) allows optimal power production and consumption and enables regional-unit energy production and consumption at the same time. For example, the use of weather data and GIS to predict renewable energy generation or to effectively manage renewable energy facilities that rely heavily on natural conditions will contribute to optimizing energy production and consumption in certain areas.

As such, the technology of the Fourth Industrial Revolution can be used to construct a convergence system centered on distributed power, as well as optimizing existing facilities

Article history:

Received (April 4, 2019), Review Result (May 30, 2019), Accepted (August 26, 2019) 
operations in the process of transition to a low-carbon energy system. The future energy system is expected to be a form of adjustment (demand response) of supply and demand balance based on regional energy self-sufficiency systems utilizing distributed energy sources (e.g. standalone microgrids, virtual power plants, etc.). In the demand management sector related to energy consumption, the fourth industrial revolution technology will strengthen ICT-based demand management, including energy-saving and demand response, and contribute to the development of new energy business models.

This concept of the Internet of Energy ( $\mathrm{IoE}$ ) is a network infrastructure that integrates energy and data/information, enabling power generation and energy storage capacity to be balanced with energy demand in real-time. The IoE will enable active integration of AMI (Advanced Metering Infrastructure), Demand-Response, e-Prosumer, electric vehicles (V2G) connected to the grid as power consumption and storage media, and various distributed power and energy storage devices and grid management.

The IoE will present a groundbreaking methodology for monitoring and communicating energy distribution, energy storage and energy grids by providing a variety of information and connectivity to the energy grids in conjunction with buildings, cars and cities. It will also leverage renewable energy, energy storage, smart meters, energy gateways, Smart Plugs and consumer electronics to provide energy consumers, manufacturers and utility providers with new and powerful tools to reduce resources and costs, and control and manage target devices.

With the IoE, energy can be delivered in both directions at any time and anywhere, and monitoring of energy consumption will be available at all levels, from individual units to regional, national and global units. It provides consumers with a reliable, flexible, efficient and economical energy supply network, allowing them to combine centralized large-scale power plants with distributed, small renewable energy sources such as solar and wind power as a single fusion system. For this purpose, a system with blockchain-based smart solutions is needed to implement energy trading in the autonomous form of the region by introducing a model of virtual power plants in the region.

This paper designs a blockchain-based power transaction process that allows individuals (producers) to produce and use power themselves or sell the remaining power to others, rather than transmitting and using the developed power from the existing centralized power.

\section{Related works}

\subsection{Blockchain for power trading}

Blockchain technology is expanding its use from the parts that can be applied to transactions that exclude intermediary agencies. It is emerging as a hot topic in various applications such as the Internet of Things and self-driving cars in financial transactions that can reduce virtual currency functions and transaction fees. In addition, various projects are underway in the energy sector and many changes in existing power trading and supply systems are expected when commercialized [4][5][6][7][8]. Blockchain-based technologies are applied to transaction systems that compensate for solar power production in a "Solar Coin," prosumer trading systems that produce solar energy and trade surplus electricity between neighbors, and electric vehicle charging stations.

A blockchain-based system for energy trading consists of three parts [1]. There is an energy trading system between EV and CS, a computer system using Edge as a service, and a blockchain-based safe energy trading mechanism using Edge as a service. 
EVs in smart cities must be charged with energy from CS placed in various locations. EV needs to exchange energy with CS in geographical locations to achieve maximum benefit in terms of energy and price. Similarly, CS sells available energy to maximize profits. However, transferring data from an EV to the cloud or server could be a higher delay and an additional cost for an energy transaction service provider. To overcome this, edge computing reduces additional delays and costs by enabling data processing and decision-making closer to end-user locations.

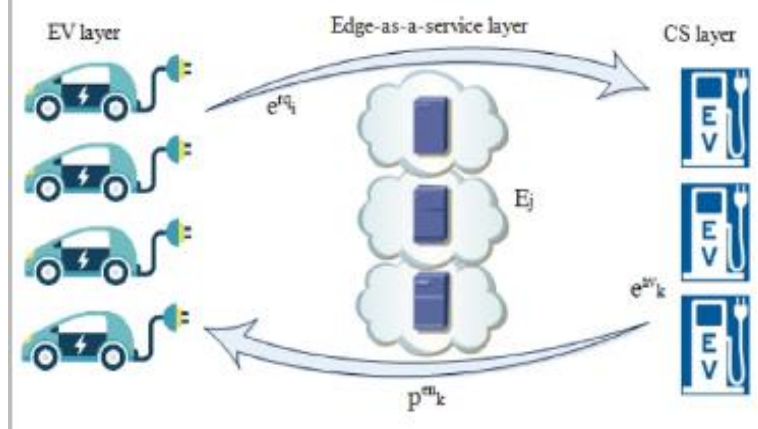

Figure 1. Energy transaction mechanism [1]

Blockchain systems are used to provide security for transactions between EV (electric vehicles) and CS (charging stations). The consensus algorithm is used to validate transactions shared between the approver nodes in the blockchain. All nodes selected as edge nodes serve as approver nodes used to calculate EV work.

(1) Transaction initialization: EV that initially wish to exchange energy with CS transmit authentication information to trusted nodes. The edge node then calculates the hash function to initialize transactions on the network.

(2) Block header generation: Once the deal for an EV is finalized, the block headers are generated by calculating the hash from the muckle hash tree.

(3) Block validation and activity proof (POW) generation: The approver node calculates PoW for each transaction to be added from the blockchain upon receipt of a message from the edge node. All edge nodes present in the network act as approver nodes to validate transactions in that EV. All approver nodes calculate PoW, and when more than $50 \%$ of the general agreement is reached, the transaction is added to the blockchain according to the result.

(4) If the value of PoW corresponds to the received message, the block shall be deemed to have been verified on the approver node. All approver nodes calculate PoW for EV and send the results to the transaction server. If you agree to the general agreement that more than $50 \%$ of the blocks on the node are valid, the blocks are added in the blockchain, otherwise, the transaction is discarded.

Meanwhile, Truc Dinh et al. [2] announced how blockchain could be used to address data privacy issues in the Internet of Things (IoT). Through smart contract, a system model with access control mechanisms was developed to allow users to fully control their data and track how third-party services access the data.

Yunru Zhang et al. [3] controlled data access using blockchain models and attribute-based encryption systems in the Internet of Things environment to protect personal information. In order to achieve granular access control, the Smart Shield was used to create a licensed access control table (PACT, Permission Access Control Table) and the owner first placed the Smart Shack on the access control table of the blockchain. 


\subsection{Smart contract}

The smart contract was first proposed by Nick Szabo in 1994. The existing contract is written and to fulfill the terms of the contract, the actual person must perform as per the contract. However, if you create a contract with a digital command, you can execute the contract automatically according to the terms.

The smart contract creates contracts for the terms of transactions between traders, monitors contract performance over a blockchain network, and can execute contracts quickly without separate verification by the central system on whether the contract is fulfilled or not by automatically executing the contract. Through smart contracts, consumers and suppliers can quickly trade and settle production and save energy, and further reduce transaction fees incurred through brokerage houses.

The smart contract operates as [Figure 2] inside the blockchain network when transactions occur. All nodes in the network will share content registration transactions and store them in the transaction database. After this step, the smart contract application is executed according to the contents of the transaction and the results are reflected in the smart contract database.
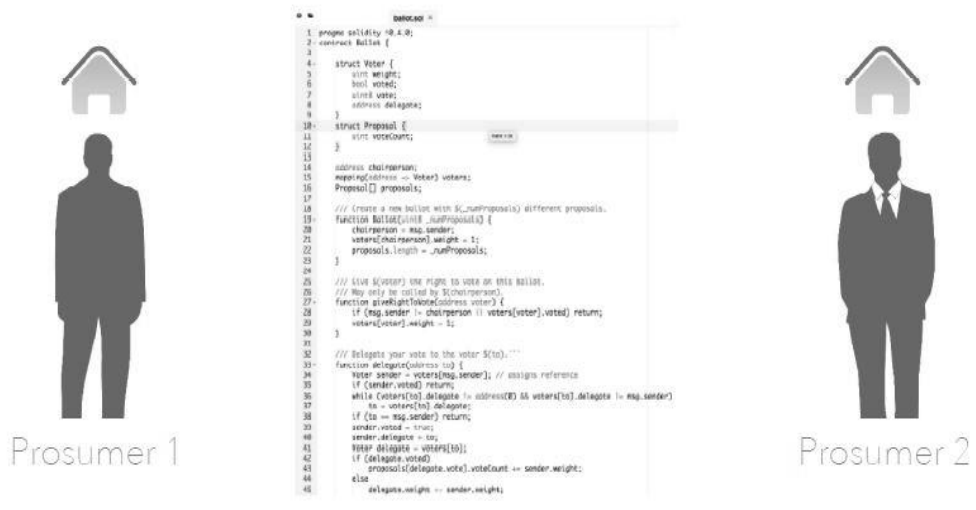

Figure 2. Smart contract

\section{Blockchain-based power trading process}

In this paper, the power trading process based on blockchain is constructed as shown in [Figure 3]. [Figure3] is largely divided into the Access device layer, blockchain layer, and Edge layer. The access device layer is a user layer that attempts to access shared data using smart devices, while the blockchain layer is configured for data access and security. Edge Layer is configured to identify the users accessing the data and provide the data.

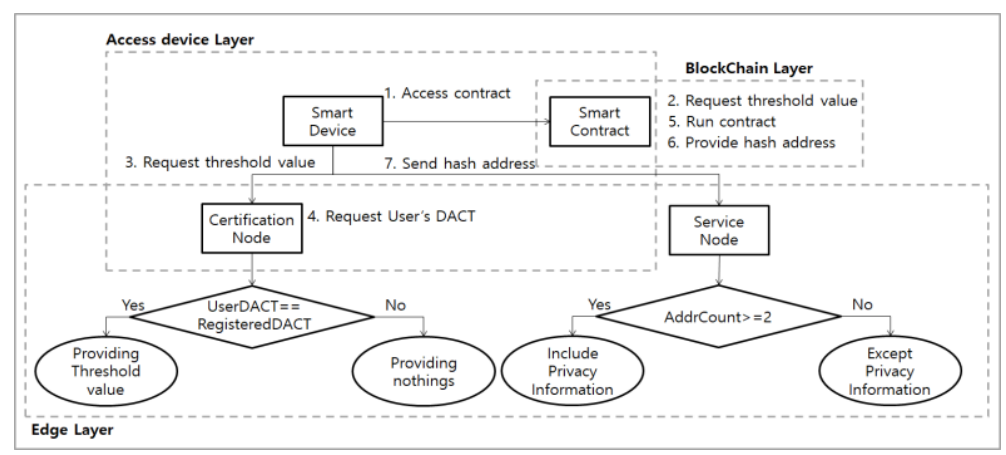

Figure 3. Blockchain-based power trading process 
(1) The user accesses the Smart Contract using the Smart Device.

(2) Smart Contract requires a threshold to operate the contract.

(3) The user shall request a threshold from the Certification Node.

(4) Certification Node requires the user to have a Dynamic Access Control Table (DACT) consisting of the identity of things (IDoT). The Certification Node matches the DACT that is delivered to the DACT that is registered by the user in advance. When matched, the threshold is given to the user.

(5) The user drives the Smart Contract using a threshold.

(6) Receive the hash address of the shared data from the Smart Contract that has been activated.

(7) Transfer the data to Service Node, which provides the shared data. The delivered Service Node shares data with the owner's privacy information if the number of hash addresses received is more than one.

The proposed system consists of a blockchain-based secure energy trading mechanism using edge-as-a-service.

\section{Conclusion}

In this paper, we worked a blockchain-based power trading model that allows individuals (producers) to produce and use power themselves or sell the remaining power to others. In other words, if prosumer1 that wants to purchase electricity through blockchain's smart contract function requests a transaction to prosumer2, which sells electricity, it generates trading information as a block, anonymously releases the trading information to participants and utilities in the power trading, which is verified and blocks with the previous trading.

\section{Acknowledgment}

This research was supported by the Basic Science Research Program through the National Research Foundation of Korea (NRF) funded by the Ministry of Education (2019R1F1A1056507).

\section{References}

[1] Anish Jindal, Gagangeet Singh Aujla, and Neeraj Kumar, "Survivor: A blockchain-based edge-as-a-service framework for secure energy trading in SDN-enabled vehicle-to-grid environment," Computer Networks, vol.153, pp.36-48, (2019) DOI: 10.1016/j.comnet.2019.02.002

[2] Truc Dinh, Trung Nguyen, and Hoang-Anh Pham, "Leveraging blockchain to enhance data privacy in IoTbased applications," 7th International Conference, Computational Data and Social Networks, pp.211-221, (2018) DOI: 10.1007/978-3-030-04648-4_18

[3] Yunru Zhang, Debiao He, and Kim-Kwang Raymond Choo, "BaDS: Blockchain-based architecture for data sharing with ABS and CP-ABE in IoT," Wireless Communications and Mobile Computing, vol.2018, Article ID 2783658, 9 pages, (2018), DOI: 10.1155/2018/2783658

[4] Hossein Shafagh, Anwar Hithnawi, and Simon Duquennoy, "Towards blockchain-based auditable storage and sharing of IoT data," CCSW '17 Proceedings of the 2017 on Cloud Computing Security Workshop, pp.45-50, (2017) DOI:10.1145/3140649.3140656

[5] Muneeb Ul Hassan and Mubashir HusainRehmani, JinjunChen, "Privacy preservation in blockchain based IoT systems: Integration issues, prospects, challenges, and future research directions," Future Generation Computer Systems, vol.97, pp.512-529, (2019) DOI: 10.1016/j.future.2019.02.060 
[6] Zhetao Li, Jiawen Kang, Rong Yu, Dongdong Ye, Qingyong Deng, and Yan Zhang, "Consortium blockchain for secure energy trading in industrial internet of things," IEEE Transactions on Industrial Informatics, vol.14, No.8, pp.3690-3700, (2018) DOI: 10.1109/TII.2017.2786307

[7] Hossein Shafagh, Anwar Hithnawi, and Simon Duquennoy, "Towards blockchain-based auditable storage and sharing of IoT data," CCSW '17 Proceedings of the 2017 on Cloud Computing Security Workshop, pp.45-50, (2017), DOI:10.1145/3140649.3140656

[8] You-jin Song, Aria Seo, Jaekyu Lee, and Yei-chang Kim, “Access control policy of data considering varying context in sensor fusion environment of internet of things," KIPS Transactions on Software and Data Engineering, vol.4, pp.409-418, (2015) DOI: 10.3745/KTSDE.2015.4.9.409 